\title{
Mildly Oxidized Graphene: Facile Synthesis, Characterization, and Application as a Matrix in MALDI Mass Spectrometry
}

\author{
Qian Liu, Mengting Cheng, and Guibin Jiang*[a]
}

\begin{abstract}
Graphene $(\mathrm{G})$ is a new allotropic member of carbon with a unique two-dimensional and one-atom-thick sheet structure. It has attracted tremendous research interest in both academic and industrial areas due to its exceptional physical and chemical properties. ${ }^{[1-3]}$ However, a serious problem in synthesis and application of $G$ is aggregation. Due to the strong hydrophobic nature of $G, G$ sheets have a strong tendency to aggregate to $G$ clusters or even restack to graphite particles through van der Waals interactions. The resulting $\mathrm{G}$ agglomerates are very hard and insoluble in water or organic solvents, making further processing difficult. Since most of the exceptional properties of $G$ can only be achieved with individual sheets, prevention of aggregation of $\mathrm{G}$ sheets is of particular importance for its applications.

Aggregation of $\mathrm{G}$ can be reduced by using stabilizers. ${ }^{[4-6]}$ However, attachment of foreign molecules onto $G$ sheets may cause an adverse impact on the applications. As an alternative, graphene oxide (GO) has an excellent water dispersibility and has been used as a substitute for $G$ in many applications. ${ }^{[3]} \mathrm{GO}$ is usually obtained by exfoliation of graphite oxide, ${ }^{[7-8]}$ which can be synthesized from graphite. ${ }^{[9]}$ However, the heavy oxidation during the synthesis can severely damage the large delocalized $\pi$-electron system of $G$ and cause many imperfections and defects in the GO sheets. Thus, the unique electronic and optical absorption properties of $\mathrm{G}$ are seriously compromised in GO. Li et al. ${ }^{[10]}$ reported the preparation of a processable aqueous dispersion of chemically converted G from GO; however, the obtained $\mathrm{G}$ dispersion was only stable under basic conditions and the reductant was difficult to remove from the dispersion. Therefore, facile and scalable methods for the synthesis of low-defect, well-dispersed, and clean $\mathrm{G}$ sheets are still urgently desired. Inspired by GO, we hypothesized that controlling the oxidation degree of $\mathrm{G}$ may provide a feasible approach for this purpose. However, although significant efforts have been made on developing synthetic strategies of
\end{abstract}

[a] Dr. Q. Liu, M. Cheng, Prof. Dr. G. Jiang

State Key Laboratory of Environmental Chemistry and Ecotoxicology

Research Center for Eco-Environmental Sciences

Chinese Academy of Sciences

Beijing 100085 (P.R. China)

Fax: (+86) 010-62849179

E-mail: gbjiang@rcees.ac.cn

Supporting information for this article is available on the WWW under http://dx.doi.org/10.1002/chem.201203791.
$\mathrm{G}$, little attention has been paid to controlling the oxidation degree of $\mathrm{G}^{\left[{ }^{[1-12]}\right.}$

Herein, we report a very simple method for the synthesis of mildly oxidized graphene by oxidizing chemically converted $G$ with diluted nitric acid (2m; Scheme 1A). We aimed to find a facile approach to prevent the aggregation of $\mathrm{G}$ sheets while maintaining the $\mathrm{G}$ structure as intact as possible. To distinguish from $\mathrm{GO}$, the produced material is called acid-oxidized graphene (AOG). The AOG showed good dispersibility in water (up to $1 \mathrm{mg} \mathrm{mL}^{-1}$ ). Meanwhile, unlike GO (Scheme 1B), the main framework of $\mathrm{G}$ was not disrupted, and thus most of the exceptional properties of $G$ can be maintained. Therefore, the AOG combines the advantages of $\mathrm{G}$ and $\mathrm{GO}$ while avoiding their disadvantages. Furthermore, the AOG contains no stabilizer molecules and can be highly pure, which is very favorable for its applications.

We found that the AOG aqueous dispersion was rather stable. Even after storing at room temperature for six months, no precipitate was observed (Figure 1A), which indicated that the electrostatic repulsion between the polar groups in AOG sheets is strong enough to prevent the aggregation of AOG sheets. Furthermore, it was found that the AOG could be dispersed in saline, acidic, and basic solutions (Figure 1A). These properties favor its applications under different conditions.

The AOG was characterized by different techniques. From AFM and TEM measurements (see Figure S1 and S2 in the Supporting Information), it was deduced that AOG consisted of ultrathin and crumpled nanosheets with no bulk agglomeration. AFM measurements showed that the nitric acid oxidization did not change the single layer nature of $G$ nanosheets, with a thickness of $1.224 \mathrm{~nm}$ (see Figure S1 in the Supporting Information). The oxidation degree of AOG was checked by XPS and FTIR spectroscopy. As shown in Figure 1B, peak fitting of the $\mathrm{C} 1 \mathrm{~s}$ bands of AOG yielded three main components at $284.4,285.5$, and $288.5 \mathrm{eV}$ assigned to $\mathrm{C}-\mathrm{C}, \mathrm{C}-\mathrm{O}$, and $\mathrm{O}-\mathrm{C}=\mathrm{O}$ bonds, respectively. ${ }^{[13]}$ The enhancement of oxygen functionalities indicated the successful oxidation of G. However, the peak intensities of oxygen functionalities of AOG were much lower than those of GO, which indicated that the oxidation degree of AOG was much weaker than GO. Furthermore, the $\mathrm{C}=\mathrm{O}$ bond was absent and the $\mathrm{O}-\mathrm{C}=\mathrm{O}$ bond was enhanced in AOG. This implies that carboxyl groups might dominate the polar groups in AOG sheets. Notably, in wide-scan XPS spectra 


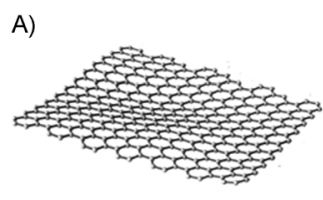

Graphene

(G)
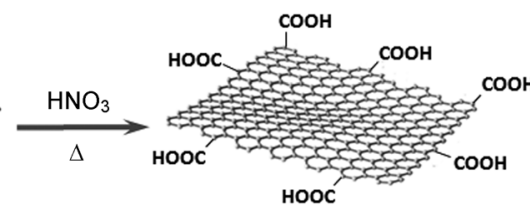

Acid-oxidized graphene (AOG)

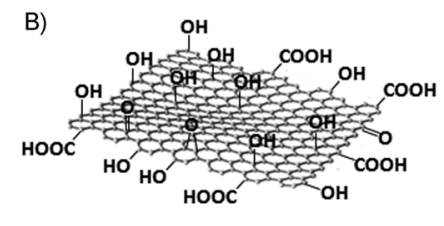

Graphene oxide (GO)

Scheme 1. A) Chemical route to the AOG and the models of G and AOG sheets. B) Model of GO sheets.

A)
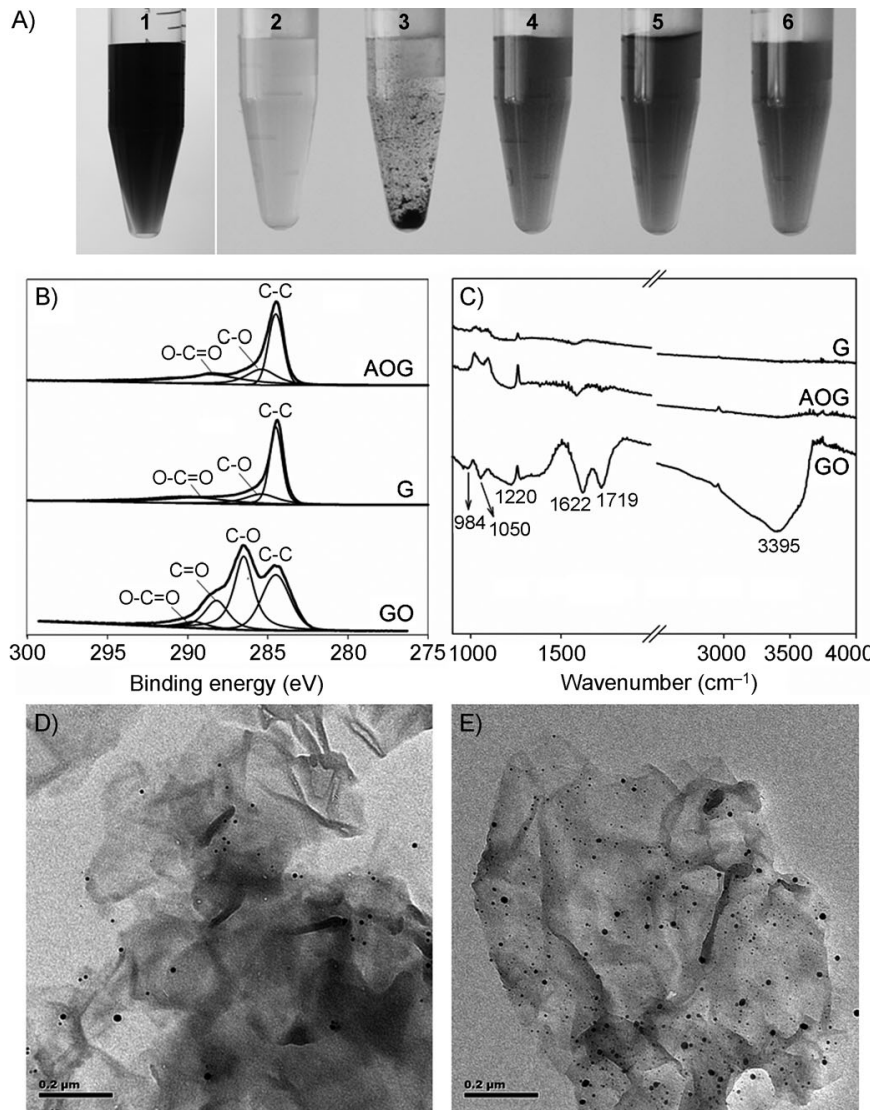

Figure 1. A) Digital photograph of 1) AOG aqueous dispersion at $100 \mu \mathrm{gL}^{-1}$ after storing at room temperature for six months and 26) aqueous dispersions at $50 \mu \mathrm{g} \mathrm{mL}^{-1}$ of $\mathrm{GO}(2), \mathrm{G}(3)$, and $\mathrm{AOG}$ in phosphate buffer saline (4), AOG at $\mathrm{pH} 3$ (5), and AOG at pH 11 (6). B) C1s XPS spectra of AOG, G, and GO. The spectrum of GO was reprinted with permission from Elsevier. ${ }^{[28]}$ C) FTIR spectra of AOG, G, and GO. D,E) TEM images of AOG (D) and GO (E) sheets decorated with CTAB-capped gold nanoparticles.

(see Figure S3 in the Supporting Information), no N1s peak was observed for AOG, which indicated that few nitrogen atoms were introduced into AOG during the oxidization process. Figure $1 \mathrm{C}$ shows the FTIR spectra of AOG, GO, and $\mathrm{G}$. GO had a strong and broad absorption at $3395 \mathrm{~cm}^{-1}$ due to the $\mathrm{O}-\mathrm{H}$ stretching vibration. The $\mathrm{C}=\mathrm{O}$ stretching of carboxyl groups was observed at $1719 \mathrm{~cm}^{-1}$, and the absorption due to the $\mathrm{O}-\mathrm{H}$ bending vibration, epoxide groups, and skeletal ring vibrations were located at $1622 \mathrm{~cm}^{-1} \cdot{ }^{[14]}$ In con- trast to GO, G showed no significant feature peaks in the FTIR spectrum probably due to the overlapping of the strong absorption of $\mathrm{G}$ sheets. For AOG, the peaks at 1719 and $1622 \mathrm{~cm}^{-1}$ were observable but not as prominent as that observed for GO, and the peak at $3395 \mathrm{~cm}^{-1}$ nearly disappeared. These observations proved that the oxidation occurred in AOG sheets but was much milder than that in GO sheets. The $\mathrm{C} / \mathrm{O}$ ratios in $\mathrm{AOG}, \mathrm{G}$, and $\mathrm{GO}$ measured by EDX were 7.4, 22.0, and 1.9, respectively (see Figure S4 in the Supporting Information). The mild oxidation of AOG was also confirmed by absorption spectrometry, Raman spectrometry, XRD, and TGA (see Figures S5-S8 in the Supporting Information).

We designed a novel nanoparticle-labeling method to determine the oxidation sites in AOG sheets. Because the oxygen-containing groups in AOG and GO sheets (mainly phenolic hydroxyl, epoxy and carboxyl groups) ${ }^{[15]}$ can be ionized and take negative charges (see Figure S9 in the Supporting Information), oppositely charged gold nanoparticles (AuNPs) can be assembled on these groups and used as an indicator for the oxidation sites. The positively charged AuNPs were prepared by using cationic surfactant cetyltrimethylammonium bromide $(\mathrm{CTAB})$ as a protectant reagent and $\mathrm{NaBH}_{4}$ as a reducing reagent. As shown in Figure 1D, for AOG, only a limited number of AuNPs were observed on the surface of the AOG sheets. Whereas for GO (Figure 1E), a large number of AuNPs distributed uniformly on the entire GO sheets. These results clearly indicate that AOG sheets have much less oxygen-containing groups than GO sheets. Therefore, the intactness of the $\mathrm{G}$ structure can be maintained to a larger degree in AOG.

To demonstrate the potential advantage of this material, we herein show the application of AOG as a matrix in matrix-assisted laser desorption/ionization mass spectrometry (MALDI MS). Use as MALDI matrix is a very interesting application aspect for $\mathrm{G}^{\left[{ }^{[16-24]}\right.}$ An ideal MALDI matrix should have strong energy absorption and transfer capability. In this regard, $G$ is a good candidate due to its fast charge carrier mobility ${ }^{[25]}$ and universal and frequency-independent optical absorption properties. Furthermore, G has efficient electron-phonon coupling and high thermal conductivity. ${ }^{[26]}$ Therefore, $\mathrm{G}$ can cause less fragmentation of analyte molecules and produce low background interference in the low-mass region. Unfortunately, the $G$ matrix also suffers from aggregation, which may significantly reduce the desorption/ionization efficiency. Therefore, the AOG was 

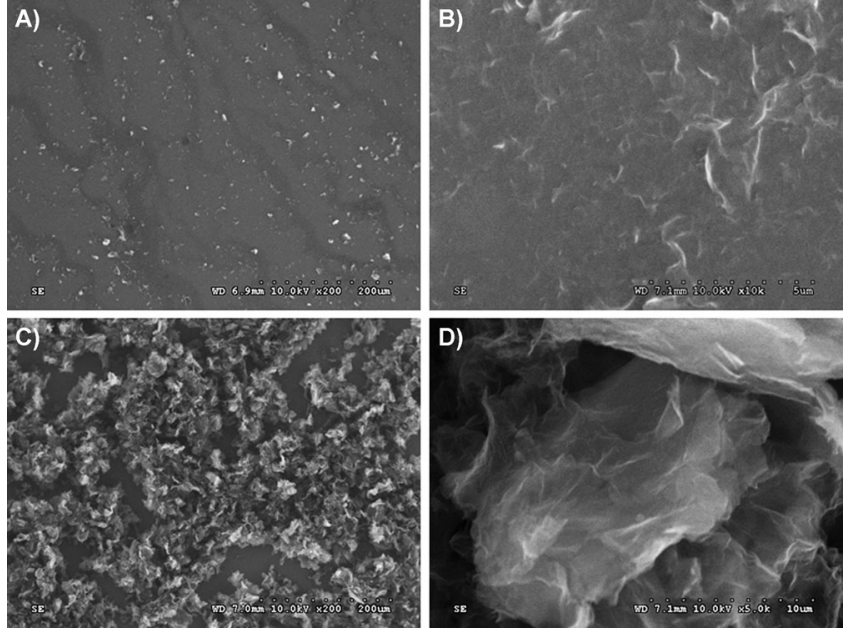

Figure 2. SEM characterization of the AOG and G matrix: A) AOG at $\times 200$, B) AOG at $\times 10000$, C) $\mathrm{G}$ at $\times 200$, and D) $\mathrm{G}$ at $\times 5000$.

expected to overcome this problem while maintaining the excellent energy absorption and transfer capability of $\mathrm{G}$ in MALDI-MS.

The AOG matrix on the MALDI target was firstly characterized by SEM. From Figure 2A, the AOG sheets could be compactly deposited on the MALDI target and the obtained AOG matrix was quite flat and homogeneous. From the high-resolution view (Figure 2B), many wrinkled textures corresponding to the folded regions of the AOG sheets could be observed, implying that the AOG sheets kept the morphology of the nanosheet in the matrix. On the contrary, the $\mathrm{G}$ matrix (Figure $2 \mathrm{C}$ ) was inhomogeneous and rugged. The $\mathrm{G}$ sheets aggregated or restacked to discrete clusters during the drying process (Figure 2D), and thereby a considerable part of the MALDI target surface was bare. For the conventional organic matrix, the analytes need to be co-crystallized with the matrix, and lead to the generation of "hot spots" in MALDI-TOF MS analysis (see Figure S10 in the Supporting Information). This may adversely affect the reproducibility.

Four types of environmental pollutants were selected as model analytes (molecular weight 266-500, see Figure 3 for chemical structure), including an organochlorine pesticide pentachlorophenol (PCP), an endocrine disrupter estradiol (E2), a brominated flame retardant 2,2',4,4'-tetrabromodiphenyl ether (BDE-47), and an ionic compound perfluorooctanesulfonic acid (PFOS). The morphology of the matrix after loading with different analytes was also studied by SEM (see Figure S11 in the Supporting Information). The matrix loaded with nonpolar analytes (PCP, E2, and BDE-47) was found to be flatter than that with PFOS, probably because non-polar molecules can assist in dispersing AOG.

The MALDI-TOF MS results are shown in Figure 3. The performance of AOG was compared to G, GO, and a conventional organic matrix, $\alpha$-cyano-4-hydroxycinnamic acid (CHCA). Overall, for all the four analytes, AOG showed the best performance among the tested matrices. For PCP (Figure 3A), multiple peaks of $[M-\mathrm{H}]^{-}$and $[M-\mathrm{H}-\mathrm{Cl}]^{-}$ were detected at $m / z: 264.1$ and 229.1 with AOG and $\mathrm{G}$ as the matrix. More importantly, the peak intensity of $[M-\mathrm{H}]^{-}$ for AOG was 3.7 times higher than that for $G$. The reason for this is attributed to the better dispersibility of the AOG matrix relative to the $\mathrm{G}$ matrix, causing higher laser energy absorption and transfer efficiency. Furthermore, introduction of some polar groups by mild oxidation in AOG sheets
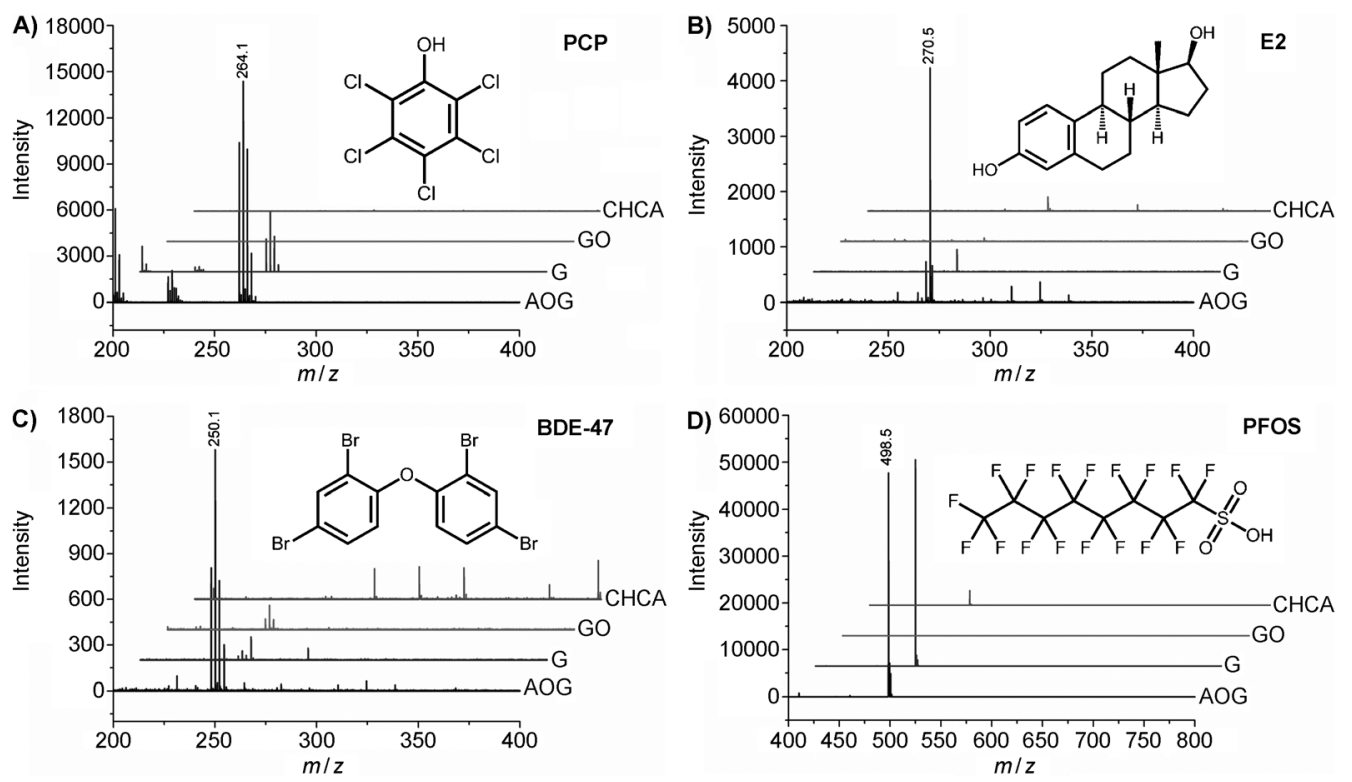

Figure 3. MALDI-TOF MS analysis of PCP (A), E2 (B), BDE-47 (C), and PFOS (D) with different matrices (AOG, G, GO, and CHCA). Analyte concentration: $50 \mu \mathrm{g} \mathrm{mL}^{-1}$. The insets show the chemical structures of the analytes. 
can also mitigate the strong hydrophobic interaction between PCP and the matrix, which is favorable for the analyte desorption. Whereas for the GO matrix, no peaks corresponding to PCP were detected, which is likely due to the imperfect electronic conjugation and reduced optical absorption capacity of GO (see Figure S3 in the Supporting Information). For CHCA, no PCP peaks were detected in either negative or positive ion mode, which suggests that the conventional organic matrix is not suitable for analysis of small molecules. Similar results were also obtained for E2 and BDE-47 (Figure 3B and C). The peak intensity of $[M-\mathrm{H}]^{-}$of E2 at $m / z: 270.5$ for AOG was 4.2 times higher than that for $\mathrm{G}$, and 70.5 times higher than that for GO. For BDE-47, triple peaks of $\left[M-\mathrm{C}_{6} \mathrm{H}_{3} \mathrm{Br}_{2}\right]^{-}$at $m / z: 250.1$ were detected for AOG, G, and $\mathrm{GO}$, and the peak intensity for AOG was 25.9 times higher than $\mathrm{G}$ and 9.8 times higher than GO. These results definitely demonstrate that the AOG is a very promising matrix for MALDI-TOF MS.

For PFOS (Figure 3D), the peak intensity of $[M-\mathrm{H}]^{-}$at $m / z: 498.5$ for AOG was 15.3 times higher than that for CHCA. Furthermore, G showed a comparable performance with AOG, probably because PFOS is very easy to ionize and desorb from the matrix, and thus the dispersing state of the matrix exerts no significant effect on the ionization/desorption efficiency. Therefore, for ionic analytes, $\mathrm{G}$ may also work well; whereas for nonpolar compounds, matrixes of higher quality, such as AOG, must be used to achieve highly efficient ionization/desorption.

High sensitivity has been achieved with the AOG matrix. The detection limits $(s / n=3)$ of PCP, E2, BDE-47, and PFOS were $60 \mathrm{fg}, 0.25 \mathrm{ng}, 0.5 \mathrm{ng}$, and $10 \mathrm{fg}$, respectively. The better homogeneity of the AOG matrix also brings in better reproducibility. For example, in analysis of BDE-47, the shot-to-shot RSD $(n=20)$ at different locations on the AOG matrix was $16.7 \%$, while this value on the $G$ matrix was $54.1 \%$. In addition, the AOG matrix showed a good tolerance for salt (see Figure S12 in the Supporting Information).

It is worth mentioning that some other methods, such as chemical vapor deposition (CVD) and epitaxial growth, can also generate a high quality of $\mathrm{G}$ films. However, these methods need complicated devices and special substrates, and the resulting films are difficult to be transferred to the MALDI target.

To summarize, mild oxidization with diluted nitric acid allows us to obtain low-defect AOG material with excellent water dispersibility on a large scale. The preparation process is very simple and environmentally friendly, and the obtained AOG is quite clean without any stabilizers. We showed the application of AOG as a matrix in MALDI MS. The AOG matrix yielded significantly higher signals than $G$, $\mathrm{GO}$, and conventional organic matrixes for nonpolar analytes. Considering the enhanced water dispersibility and lowoxidation and -defect structure of AOG, we strongly believe that this material has great potential for applications in other fields.

\section{Experimental Section}

Preparation: AOG was synthesized by refluxing the chemically converted $\mathrm{G}$ sheets in $2 \mathrm{M}^{\mathrm{HNO}_{3}}$ (Merck, Darmstadt, Germany) at $105^{\circ} \mathrm{C}$ for $24 \mathrm{~h}$, followed by washing with water several times. The product was dispersed in water at $1 \mathrm{mg} \mathrm{mL}^{-1}$ and sonicated for $2 \mathrm{~h}$ to give a stable aqueous dispersion. GO was synthesized by using a modified Hummers method as described in previous reports. ${ }^{[7-8,27]}$ The AOG and GO dispersions were centrifuged at $12000 \mathrm{rpm}$ for $10 \mathrm{~min}$ to remove the unexfoliated content. The supernatants were used for the following experiments and the concentration of AOG or GO was determined by UV/Vis absorption spectrometry. Other experimental details are given in the Supporting Information.

\section{Acknowledgements}

The authors acknowledge the financial support from the National Basic Research Program of China (no. 2009CB421605), the National Natural Science Foundation of China (no. 21107120, 20921063, 20931160427), and the State Key Laboratory of Chemo/Biosensing and Chemometrics (no. 2010-2).

Keywords: aggregation $\cdot$ graphene $\cdot$ MALDI MS • matrix • oxidation

[1] A. K. Geim, K. S. Novoselov, Nat. Mater. 2007, 6, 183-191.

[2] C. N. R. Rao, A. K. Sood, K. S. Subrahmanyam, A. Govindaraj, Angew. Chem. 2009, 121, 7890-7916; Angew. Chem. Int. Ed. 2009, $48,7752-7777$.

[3] Y. W. Zhu, S. Murali, W. W. Cai, X. S. Li, J. W. Suk, J. R. Potts, R. S. Ruoff, Adv. Mater. 2010, 22, 3906-3924.

[4] S. Das, A. S. Wajid, J. L. Shelburne, Y. C. Liao, M. J. Green, ACS Appl. Mater. Interfaces 2011, 3, 1844-1851.

[5] X. H. An, T. J. Simmons, R. Shah, C. Wolfe, K. M. Lewis, M. Washington, S. K. Nayak, S. Talapatra, S. Kar, Nano Lett. 2010, 10, $4295-$ 4301.

[6] M. Lotya, P. J. King, U. Khan, S. De, J. N. Coleman, ACS Nano 2010, 4, 3155-3162.

[7] S. Stankovich, D. A. Dikin, G. H. B. Dommett, K. M. Kohlhaas, E. J. Zimney, E. A. Stach, R. D. Piner, S. T. Nguyen, R. S. Ruoff, Nature 2006, 442, 282-286.

[8] S. Stankovich, R. D. Piner, X. Chen, N. Wu, S. T. Nguyen, R. S. Ruoff, J. Mater. Chem. 2006, 16, 155-158.

[9] W. S. Hummers, R. E. Offeman, J. Am. Chem. Soc. 1958, 80, 1339.

[10] D. Li, M. B. Muller, S. Gilje, R. B. Kaner, G. G. Wallace, Nat. Nanotechnol. 2008, 3, 101-105.

[11] Y. X. Xu, K. X. Sheng, C. Li, G. Q. Shi, J. Mater. Chem. 2011, 21, 7376-7380.

[12] G. Eda, J. Ball, C. Mattevi, M. Acik, L. Artiglia, G. Granozzi, Y. Chabal, T. D. Anthopoulos, M. Chhowalla, J. Mater. Chem. 2011, 21, 11217-11223.

[13] Y. Wang, Y. Y. Shao, D. W. Matson, J. H. Li, Y. H. Lin, ACS Nano 2010, 4, 1790-1798.

[14] C. Y. Hou, Q. H. Zhang, M. F. Zhu, Y. G. Li, H. Z. Wang, Carbon 2011, 49, 47-53.

[15] O. C. Compton, S. T. Nguyen, Small 2010, 6, 711-723.

[16] Q. Liu, J. B. Shi, G. B. Jiang, TrAC Trends Anal. Chem. 2012, 37, 1 11.

[17] X. L. Dong, J. S. Cheng, J. H. Li, Y. S. Wang, Anal. Chem. 2010, 82, $6208-6214$.

[18] L. A. L. Tang, J. Z. Wang, K. P. Loh, J. Am. Chem. Soc. 2010, 132, 10976-10977. 
[19] J. Zhang, X. L. Dong, J. S. Cheng, J. H. Li, Y. S. Wang, J. Am. Soc. Mass Spectrom. 2011, 22, 1294-1298.

[20] Y. Liu, J. Y. Liu, C. H. Deng, X. M. Zhang, Rapid Commun. Mass Spectrom. 2011, 25, 3223-3234.

[21] M. H. Lu, Y. Q. Lai, G. N. Chen, Z. W. Cai, Anal. Chem. 2011, 83, $3161-3169$.

[22] C. Y. Shi, J. R. Meng, C. H. Deng, Chem. Commun. 2012, 48, $2418-$ 2420.

[23] X. Z. Zhou, Y. Y. Wei, Q. Y. He, F. Boey, Q. C. Zhang, H. Zhang, Chem. Commun. 2010, 46, 6974-6976.

[24] C. Y. Shi, J. R. Meng, C. H. Deng, J. Mater. Chem. 2012, 22, $20778-$ 20785.
[25] K. I. Bolotin, K. J. Sikes, Z. Jiang, M. Klima, G. Fudenberg, J. Hone P. Kim, H. L. Stormer, Solid State Commun. 2008, 146, 351-355.

[26] A. A. Balandin, S. Ghosh, W. Z. Bao, I. Calizo, D. Teweldebrhan, F. Miao, C. N. Lau, Nano Lett. 2008, 8, 902-907.

[27] Y. X. Xu, H. Bai, G. W. Lu, C. Li, G. Q. Shi, J. Am. Chem. Soc. 2008, 130, 5856-5857.

[28] Q. Liu, J. B. Shi, L. X. Zeng, T. Wang, Y. Q. Cai, G. B. Jiang, J. Chromatogr. A 2011, 1218, 197-204.

Received: October 24, 2012

Revised: February 20, 2013 Published online: March 12, 2013 